\title{
An Unexpected Delayed Complication of Cataract Surgery: Retinal Detachment Secondary to Capsular Tension Ring Dislocation
}

\author{
Hande Taylan Şekeroğlu Elif Erdem Kemal Yar \\ Nihal Demircan \\ Ophthalmology Department, Cukurova University, Faculty of Medicine \\ Adana, Turkey
}

\section{Key Words}

Cataract - Capsular tension ring - Retinal tear - Retinal detachment

\begin{abstract}
We aim to present a case of retinal detachment secondary to capsular tension ring dislocation following cataract surgery. A 45-year-old man who underwent cataract surgery 2 years previously presented with decreased vision in his left eye. The patient's posterior capsule was intact with a well-located posterior chamber intraocular lens. Fundus examination revealed retinal detachment with retinal tears, and a capsular tension ring located around the tear was noticed during pars plana vitrectomy; it was removed through the sclerotomy site. In conclusion, dislocation of the capsular tension ring is a rare and unexpected complication of cataract surgery. Although the integrity of the posterior lens capsule is partially preserved, the ring may shift through a small tear in the bag even during its placement, and may cause retinal tears, retinal detachment or just remain silently suspended in the vitreous.
\end{abstract}

\section{Introduction}

Capsular tension rings are used to stabilize the capsular bag, reduce the tension on zonules and to provide safer conditions for phacoemulsification in patients, especially those with pseudoexfoliation or trauma-induced breakdown in zonular support. We present here an unusual case of retinal detachment secondary to a capsular tension ring-induced retinal tear.

Hande Taylan Sekeroglu, MD, FEBO Ophthalmology Department 


\section{Case Report}

A 45-year-old man who had undergone cataract surgery elsewhere 2 years previously on the left eye presented to our clinic complaining of decreased vision of 2 months' duration in the same eye. The patient was treated and followed up at the Ophthalmology Department of Cukurova University. Informed consent and institutional board approval were obtained.

Ophthalmological evaluation was completely normal for his right eye. Visual acuity was at hand motion level and the intraocular pressure was $14 \mathrm{~mm} \mathrm{Hg}$ in the left eye. Slit lamp examination showed a well-located posterior chamber intraocular lens and intact posterior lens capsule. Fundus examination of the left eye revealed retinal detachment. The retinal periphery was not optimally visualized because of peripheral posterior capsular opacification, but showed multiple retinal tears with the largest one located between 3 and 5 o'clock. Ultrasonography of the left eye showed retinal detachment as well as the large retinal tear (fig. 1). A pars plana vitrectomy was planned. Fundus examination during 20-gauge vitrectomy showed a large retinal tear located between 3 and 5 o'clock and multiple tears located peripherally, and also a dislocated capsular tension ring around the tear. One end of the ring was found under the retina just next to the large tear at the 3 o'clock position. The ring was freed from all vitreous adhesions, divided into 2 pieces with scissors and removed using vitrectomy forceps through the sclerotomy site. Barrier photocoagulation was done around the tear, and the vitrectomy was terminated after perfluorocarbon-silicone oil exchange.

On the first postoperative day, visual acuity in the left eye was 20/200. During follow-up, intraocular pressure in the left eye increased and was controlled with the use of topical brimonidine and fixed dorzolamide/timolol combination.

\section{Discussion}

Capsular tension rings are mainly preferred to maintain the contour of the capsular bag in patients who have capsular instability in complicated cataracts. Tribus et al. [1] indicated in their large series including 9,528 cataract surgeries that the frequency of capsular tension ring use was $0.7 \%$. The primary indications for the ring were found to be as mature cataract, traumatic cataract, pseudoexfoliation syndrome and lens subluxation due to Marfan syndrome. In the present case, zonular weakness and phacodonesis probably led to the use of a tension ring, and dislocation of the capsular tension ring was thought to have occurred during its placement through a possible site of capsular tear; however, the dislocation could have been caused by a late capsular contraction which resulted in capsular shrinkage and ring displacement. Another possible explanation is the misplacement of the ring in the sulcus during cataract surgery.

Possible complications of intraocular lens dislocation are retinal tears, retinal detachment, cystoid macular edema and vitreous hemorrhages [2]. Spontaneous in-thebag intraocular lens dislocations have also been reported in the literature [3-6]. The main causes were found to be associated with pseudoexfoliation and possible eye trauma. Removal of the ring has been achieved through sclerotomy sites or limbal incisions $[7,8]$.

Levy et al. [9] described a case of retinal detachment which occurred after removal of a dislocated capsular tension ring with pars plana vitrectomy. The present case differs from this case by the presence of an accompanying retinal detachment at presentation. 
To the best of our knowledge, this is the first case of isolated dislocation of a capsular tension ring into the vitreous, which possibly led to a retinal detachment with a large tear, while the posterior chamber lens was safely located in the capsular bag with an intact posterior lens capsule. The exact mechanism of the formation of the tear by capsular ring is controversial. The posterior capsule was thought to be intact during slit lamp examination before vitrectomy, but the integrity of the entire capsular bag could have been impaired by a small tear located at the equatorial region. The tear may have been created by the tension ring itself. The ring may have been placed in the sulcus or fallen into the vitreous through a tear. In the present case, the tear was considered to have occurred secondary to ring dislocation because of the critical localization of the ring around the tear area. Whatever the underlying mechanism is, it should be kept in mind that the application of a capsular tension ring requires experience and a careful follow-up because it may be associated with unexpected and very rare posterior segment complications.

\section{Disclosure Statement}

The authors have no financial interest to declare.

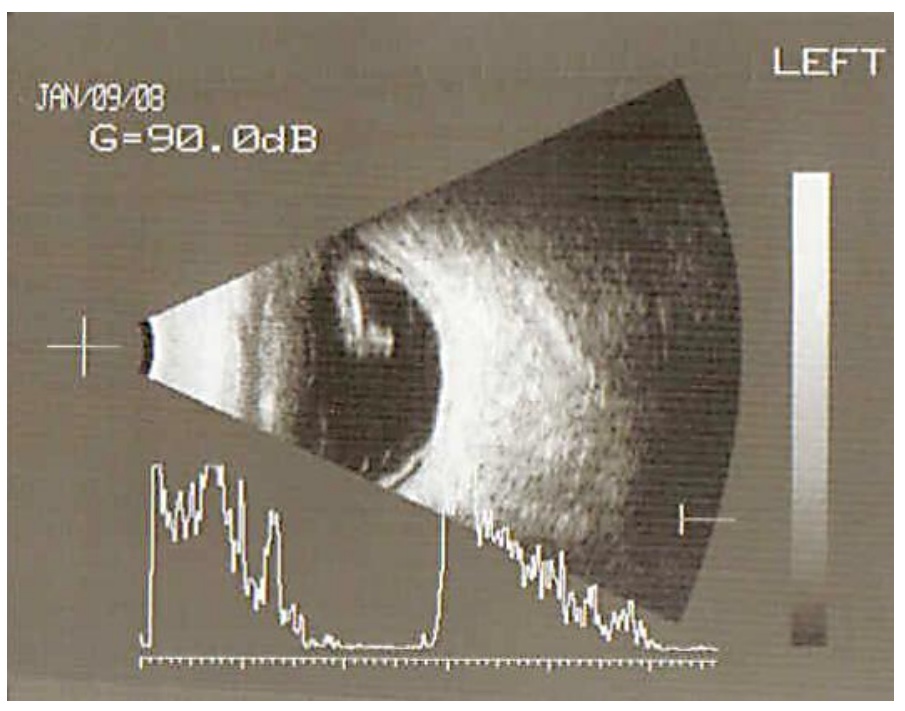

Fig. 1. B-scan ultrasonography of the left eye shows retinal detachment and a large tear. 


\section{References}

1 Tribus C, Alge CS, Haritoglou C, Lackerbauer C, Kampik A, Mueller A, Priglinger SG: Indications and clinical outcome of capsular tension ring (CTR) implantation: a review of 9,528 cataract surgeries. Clin Ophthalmol 2007;1:65-69.

-2 Shakin EP, Carty JB Jr: Clinical management of posterior chamber intraocular lens implants dislocated to the vitreous cavity. Ophthalmic Surg Lasers 1995;26:529-534.

-3 Jehan FS, Mamalis N, Crandall AS: Spontaneous late dislocation of intraocular lens within the capsular bag in pseudoexfoliation patients. Ophthalmology 2001;108:1727-1731.

-4 Masket S, Osher R: Late complications with intraocular lens dislocation after capsulorhexis in pseudoexfoliation syndrome. J Cataract Refract Surg 2002;28:1481-1484.

5 Shigeeda T, Nagahara M, Kato S, Kunimatsu S, Kaji Y, Tanaka S, et al: Spontaneous posterior dislocation of intraocular lenses fixated in the capsular bag. J Cataract Refract Surg 2002;28:1689-1693.

-6 Gross JG, Kokame GT, Weinberg DV, Dislocated In-The-Bag Intraocular Lens Study Group: In-the-bag intraocular lens dislocation. Am J Ophthalmol 2004;137:630-635.

7 Lim MC, Jap AH, Wong EY: Surgical management of late dislocated lens capsular bag with intraocular lens and endocapsular tension ring. J Cataract Refract Surg 2006;32:533-535.

8 Lang Y, Fineberg E, Garzozi HJ: Vitrectomy to remove a posteriorly dislocated endocapsular tension ring. J Cataract Refract Surg 2001;27:474-476.

-9 Levy J, Klemperer I, Lifshitz T: Posteriorly dislocated capsular tension ring. Ophthalmic Surg Lasers Imaging 2005;36:416-418. 